Artigo

\title{
Sujetos y cuerpos asistidos \\ Un análisis de la reproducción asistida en el Río de la Plata
}

\author{
Assisted subjects and bodies \\ An analysis of assisted reproduction in the Rio de la Plata
}

Mariana Viera Cherro*

\begin{abstract}
Resumen: La implementación de las tecnologías de reproducción asistida (TRA) en el Río de la Plata y los sentidos culturales que sostienen este proceso abalan la reproducción de ciertos sujetos mientras niegan o limitan las posibilidades reproductivas de otros. En tal distinción resulta esencial la manera en que tales tecnologías definen lo que forma o no parte de la naturaleza. En este artículo analizo, mediante entrevistas a especialistas en TRA y mujeres que han transitado diversos tratamientos, las formas de dominación que sobre la dimensión corporal impone el desarrollo de las TRA en el Río de la Plata y los sentidos culturales que sostienen este proceso. Concluyo que existe un orden fuertemente heterocentrado en la práctica de la medicina reproductiva rioplatense además de una orientación a intervenir urgente y específicamente el cuerpo femenino.

Palabras clave: Tecnologías de reproducción asistida. Cultura. Género. Biopoder.

Abstract: The implementation of assisted reproductive technologies in the Rio de la Plata and the cultural senses that underlie this process, allow the reproduction of certain subjects while deny or limit the reproductive potential of others. To distinct which one can be elective for procedures, technologies play an important role in defining what is natural and what is not. In this paper I describe the forms of domination on body dimension that the development of assisted reproductive technologies in the Rio de la Plata imposes to women and which are the cultural bases that support this process. This goal is achieved using interviews with specialists in TRA and also with women who have gone through different treatments. It can be concluded that there is a strong heterocentered order in the practice of rioplatense reproductive medicine in addition to a guidance to take control urgently and specifically to the female body.
\end{abstract}

Keywords: Assisted Reproductive Technologies. Culture. Gender. Biopower.

\footnotetext{
* Licenciada en Antropología Social por la Facultad de Humanidades y Ciencias de la Educación da Universidad de la República (Udelar, Montevideo, Uruguay) es profesora adjunta del Departamento de Trabajo Social de la Facultad de Ciencias Sociales de la Udelar y profesora asistente de la Sección de Género del Centro de Estudios Interdisciplinarios Latinoamericanos de la Facultad de Humanidades y Ciencias de la Educación de la misma universidad <marianaviera@yahoo.com>.
}

Civitas, Porto Alegre, v. 15 , n. 2, p. $350-368$, abr.-jun. 2015 


\section{TRA: recorrido conceptual e histórico}

La introducción de un saber tecno científico como las tecnologías de reproducción asistida impacta en el tejido cultural tensando las redes de significación en las cuales los seres humanos estamos insertos y que a partir de nuestra actuación reproducimos y/o interpelamos (Geertz, 1996). Mujeres o parejas no podrían plantearse dar solución a un problema de fertilidad buscando un hijo biológicamente vinculado si las TRA no existieran pero al mismo tiempo no habría habido interés en desarrollar tales biotecnologías si no comenzara a plantearse la ausencia de descendencia como un problema para cuya solución se privilegiara el vínculo biológico y no otras maneras simbólicas de establecer la filiación, como la adopción.

El avance biotecnológico y las transformaciones en la dimensión cultural interactúan de forma mutua, aunque en sociedades altamente medicalizadas como las rioplatenses (Barrán, 1993), el "bio-poder" (Foucault, 2002) ejerce una importante determinación sobre tales transformaciones.

Si bien existen diversos términos para referirse a las tecnologías desarrolladas y empleadas en el campo reproductivo opto por utilizar el concepto de tecnologías de reproducción asistida en tanto enfatiza la intervención médica en el acto reproductivo y concomitantemente el lugar preponderante del poder médico. Al mismo tiempo me intereso por investigar cómo quienes se intervienen para lograr una hijo biológicamente vinculado (Diniz y Gómez Costa, 2006) interpelan o reproducen el discurso médico.

Los tratamientos de reproducción asistida se dividen en baja y alta complejidad. La baja complejidad comprende la inseminación artificial mientras las técnicas de mayor complejidad incluyen la fertilización in vitro, la transferencia de gametos a la Trompa de Falopio; la transferencia de embriones a la Trompa de Falopio y la inyección intracitoplasmática de espermatozoides en óvulos. ${ }^{1}$

En el Río de la Plata las TRA penetran primero en Buenos Aires y luego en Uruguay. La totalidad de los y las especialistas entrevistados/as en ambos países han tenido en sus trayectorias profesionales una instancia de formación en Estados Unidos o en España, en donde aprendieron las formas de trabajo. De allí provienen además los médicos y los centros que certifican la actuación de los rioplatenses.

En Argentina la reproducción asistida comienza con Roberto Nicholson, quien funda el Centro de Estudios en Ginecología y Reproducción (CEGyR)

\footnotetext{
${ }^{1}$ Por detalles de los procedimientos OMS (2010).
} 
obteniendo desde 1985 los primeros nacimientos mediante fertilización in vitro. En Uruguay el pionero de la reproducción asistida fue Gerardo Bossano quien desarrolló su actividad en el Centro de Esterilidad Montevideo (CEM) a partir de 1987 logrando el primer nacimiento por fertilización in vitro el 20 de marzo de 1990.

El desarrollo de las TRA alcanza actualmente tecnologías muy sofisticadas. Veamos en qué sentido se orientan tales avances y cuáles son las transformaciones en la red de significación cultural que conllevan, pero antes quisiera hacer una breve digresión para explicar la estrategia metodológica de la investigación en la que se enmarca el presente artículo.

\section{Apuntes metodológicos}

La investigación se propuso un abordaje etnográfico, destinado a construir la inteligibilidad del mundo social desde los propios sujetos (Althabe, 2006) La técnica empleada fue fundamentalmente la entrevista semiestructurada. La estrategia de las entrevistas a las mujeres estuvo centrada en seguir sus trayectorias sexuales y reproductivas para dar cierta naturalidad a la concatenación de hechos. En el caso de los y las especialistas se eligió estructurar la conversación según la división que ellos realizan del tipo de intervenciones: baja y alta complejidad.

Mi unidad de análisis no lo constituyeron las mujeres sino sus trayectorias para lograr el hijo biológicamente vinculado (Diniz y Gómez Costa, 2006). Casos y clínicas conforman una muestra significativa en tanto permiten abordar un objeto de análisis teórico (Guber, 2004) desde diferentes y relevantes lugares del entramado socio cultural.

Una de las mujeres entrevistadas, Sandra, es madre de dos hijos por reproducción in vitro. Se realizó los tratamientos a fines de 1990. El segundo de sus hijos fue gestado a partir de un embrión congelado sobrante del tratamiento a partir del cual logró su primer embarazo. Además tiene un tercer hijo que se gestó sin que mediara tratamiento alguno y tuvo otros dos embarazos que finalizaron en abortos, el primero inducido, antes de tener a su primer hijo y el segundo espontáneo entre el segundo y tercer hijo.

Otra entrevistada es Milagros. Tiene 35 años, está casada hace $10 \mathrm{y}$ tiene un varón de 2 años y una beba de 6 meses, ambos fueron gestados sin intervención tecnológica luego que Milagros y su pareja realizaran una inseminación y dos in vitro en Uruguay, en la primera de la cuales Milagros quedó embarazada pero abortó a dos meses de gestación, y un tercer tratamiento in vitro en Argentina. Milagros es la única de las entrevistadas que se declara católica practicante, las demás profesan la religión católica pero sin ser activas 
practicantes o se consideran agnósticas. Ninguna pertenece a una religión fuera del catolicismo.

Juliana, de 40 años al momento de la entrevista, tiene una bebé de 10 meses producto de una in vitro luego de una inseminación infructuosa. Esto con su actual pareja. Pero además tiene una hija de 12 años con una pareja anterior fruto de un embarazo en el cual no medió tecnología alguna, pero que además se logró de manera muy rápida, según recalcó la entrevistada. Esto hizo que las dificultades para lograr un embarazo con su actual pareja fueran vividas con mayor ansiedad.

Laura estaba embarazada al momento de la entrevista y tuvo mellizos en febrero de 2012; se embarazó luego del quinto tratamiento de inseminación artificial.

Camila, al igual que Laura, tiene mellizas pero producto de un tratamiento de alta complejidad. Antes se realizó cuatro inseminaciones que no dieron el resultado previsto. El embarazo múltiple sobrevino en la tercera in vitro. Producto del tratamiento del cual nacieron las mellizas quedó un tercer embrión que aún permanece congelado.

Jazmín tiene mellizos, varón y mujer, producto de una in vitro realizada hace ocho años con embriones sobrantes de un tratamiento in vitro que no prosperó y que Jazmín y su esposo habían realizado luego de un procedimiento de baja complejidad. Fue el primer caso en Uruguay de un embarazo múltiple con embriones congelados. A los nueve meses de nacidos los mellizos Jazmín se embarazó de su tercer hijo sin intervención tecnológica mediante.

Nirvana se ha realizado varias inseminaciones que no prosperaron. Con su pareja no quisieron realizarse tratamientos in vitro. Ella ha decidido abandonar la posibilidad de ser madre biológica.

Andrea, por su parte, hace diez años que se realiza tratamientos de reproducción asistida, incluyendo dos inseminaciones artificiales en una de las cuales ofició de donante de óvulos de otra mujer que tuvo un niño o niña con su óvulo. En ese segundo tratamiento ella quedó embarazada pero le tuvieron que realizar un legrado. Actualmente está en proceso de adopción.

Marcia tiene 35. Comenzó sus tratamientos con una in vitro, luego se realizó otra y un mes antes de la entrevista un tratamiento de reproducción asistida. El embarazo que abortó se produjo antes de este último tratamiento. El límite a las intervenciones para Marcia y su pareja está en la utilización de materiales genéticos de terceros; su esposo no acepta tener un hijo con óvulos de otra mujer.

Catherina se ha hecho tratamientos en Montevideo y Buenos Aires sin lograr embarazarse. Las razones obedecen a problemas que al comienzo 
parecían ser solo de su esposo pero que, debido a la baja respuesta de los tratamientos solo podrían explicarse, según los médicos, por un factor mixto.

Leticia recibió óvulos de otra mujer como parte de un tratamiento realizado en Salud Pública y al momento de la entrevista tenía mellizas de un año y medio gracias a esta in vitro a sus 42 años. Años antes se practicó un aborto voluntario.

La historia de Ingrid por su parte, aparece en el contexto de las demás entrevistas como un tanto peculiar: ella tuvo cuatrillizas cuando recién comenzaba a desarrollarse en Uruguay la fertilización asistida pero no llegó a hacerse ningún tipo de inseminación. Le recetaron medicación para estimular la producción de ovocitos ${ }^{2} \mathrm{y}$ tomando la medicación quedó embarazada.

Todas las entrevistadas tienen formación secundaria; algunas también se desarrollan en tareas profesionales con educación terciaria, como Nirvana, Marcia, Laura, Juliana, Sandra, Camila y Catherina. Leticia, Sabrina e Ingrid desarrollan tareas administrativas. Jazmín tiene formación terciaria no universitaria y trabaja de manera independiente, al igual que Milagros. La mayoría pertenecen a estratos socio-económicos medio y medio alto. Jazmín pertenece a un estrato económico alto y Leticia, por su parte, a un estrato socio económico carenciado. Se pudo realizar el procedimiento in vitro en la Clínica que funcionó en la Ciudad de Las Piedras en convenio con ASSE. ${ }^{3}$ Andrea, por su parte, habiendo tenido un pasado incluso de penurias económicas hoy goza de una condición social y económica que permite ubicarla en un estrato socio económico medio. ${ }^{4}$ Todas ellas son blancas excepto Catherina que es afrodescendiente.

Al grupo de mujeres entrevistadas heterosexuales sumé una pareja de mujeres homosexuales, Daniela y Sabrina, quienes al momento de la entrevista esperaban un hijo gestado por Sabrina y producto de una inseminación artificial realizada en Buenos Aires. Lo tomé como caso testigo en tanto me permitía iluminar zonas relativas a los procesos de reproducción asistida que transitaban las parejas heterosexuales gracias al contraste con la experiencia de personas para quienes la infertilidad no resultaba de una situación definida como médica.

\footnotetext{
${ }^{2}$ Célula sexual, o gameto, femenina (OMS, 2010).

${ }^{3}$ Durante dos años, de 2009 a 2011, se llevó adelante un proyecto en convenio con la Administración de los Servicios de Salud del Estado (ASSE) mediante el cual realizaron diversos tipos de tratamientos de reproducción humana de manera gratuita a todas aquellas parejas que tuvieran cobertura de salud pública. Esta clínica se montó en la Ciudad de Las Piedras -departamento de Canelones, vecino a la capital del país.

${ }^{4}$ Para definir la condición socio económica se tomaron en cuenta fundamentalmente la ocupación de ellas y sus parejas y el lugar donde viven.
} 
En cuanto a los y las especialistas entrevistados, en Uruguay existen únicamente tres clínicas privadas dedicadas a reproducción asistida; dos de ellas trabajan en Montevideo, la capital del país, mientras la tercera comenzó instalándose en Salto (al Norte) y ahora trabaja en Salto y también en la capital. Entrevisté a un/a representante de cada una de estas clínicas. También se entrevistó a una médica de la clínica de reproducción humana del Hospital Pereira Rossell, hospital público y universitario pero que no brinda tratamientos de alta complejidad.

La otra manera de acceder a TRA en Uruguay es a través de los centros médicos que forman parte del Sistema Nacional Integrado de Salud. ${ }^{5}$ Sin embargo estos centros no cubren los costos de todo el tratamiento 6 por lo que algunas parejas deben completarlo de manera privada. Una médica y un médico entrevistados para esta investigación se desempeñan en este tipo de centros; más allá que realicen actividad privada esta es su principal forma de acceso a quienes buscan un tratamiento de reproducción asistida.

Entrevisté en total seis especialistas en Uruguay, tres varones y tres mujeres, que se entiende representan este abanico de posibilidades en materia de reproducción humana asistida, y tres en Buenos Aires, todos ellos varones.

Estos tres centros argentinos resultan significativos por diversos motivos. Uno de ellos es el precursor de la medicina reproductiva en ese país, CEGyR, fundado por Nicholson. En otro de los centros, Fecunditas, trabaja actualmente uno de los biólogos que se formó con Nicholson y que además es actualmente uno de los directores de la Sociedad Argentina de Reproducción Asistida. El tercero, Halitus, utiliza tratamientos no tradicionales, entre ellos la transferencia de embriones en procesos de alta complejidad con anestesia local y no general, como el resto de los centros. El trabajo de campo en Buenos Aires se complementó con una publicación ${ }^{7}$ que recoge el debate dado en el marco de un seminario realizado en Buenos Aires donde participaron especialistas de clínicas de todo el país.

Entiendo que la forma como se desarrollan e implementan las biotecnologías no es neutral al género (Mayobre, s. d.). Y esta no neutralidad, que produce y reproduce formas de dominación masculinas, va más allá del sexo

\footnotetext{
${ }^{5}$ Me referiré a ellos como Instituciones Mutuales.

${ }^{6}$ Esto está cambiando a partir de la reglamentación de la ley sobre Técnicas de Reproducción Humana Asistida, reglamentación actualmente en proceso. Texto completo de la ley: $<w w w$. parlamento.gub.uy/textosaprobados/AccesoTextoAprobado.asp?Url=/textosaprobados/ camara/d20121009-003614-0524.htm >.

7 "Aspectos éticos y legales de las técnicas de reproducción asistida y las diferentes alternativas terapéuticas". Versión escrita del Simposio realizado en el Hotel Emperador de Buenos Aires los días 29 y 30 de abril de 2011.
} 
del especialista que lleve adelante los tratamientos. Por ello no se considera la diferencia sexual de los mismos en el análisis.

\section{Las situaciones naturales para la intervención de las TRA}

Como señalaba antes, la introducción de las TRA genera transformaciones en la dimensión de la cultura, obligando a revisar y eventualmente reconceptualizar los significados en torno a la reproducción en su vínculo con la sexualidad, así como a definir en relación a qué arreglos sexuales y situaciones reproductivas las TRA están llamadas a intervenir.

Coincido con varias autoras (Ramírez-Gálvez, 2005; Tamanini, 2009) en la productividad de la noción de infertilidad, tal como es definida por la medicina reproductiva, para delimitar como situaciones naturales para la intervención aquellas que respaldan, tanto en términos de significación cultural como en prácticas concretas, la existencia de un orden sexo genérico heteronormativo.

Médica A: [...] ¿qué es estéril? Que no puede tener hijos, y ¿cuáles son los que no pueden tener hijos? [...] una mujer que le sacaste el útero, ¿es estéril, si tiene óvulos y podría tener hijos en el útero de otra mujer? [...] Lo podríamos utilizar para el varón que no tiene espermatozoides, que no tiene, no solo que no eyacula. Que le abrís el testículo al medio y que le buscaste y no encontrás espermatozoide, porque hoy en día le encontrás un espermatozoide en un testículo y podés hacer la inseminación. (Uruguay, Institución Mutual)

Los avances tecnocientíficos parecen sugerir que ya no hay lugar para la esterilidad, pero ¿qué diferencia a esta noción de la de infertilidad? Contraria a la esterilidad, la noción de infertilidad define la existencia de algunas limitantes para la intervención de las TRA, ¿en qué orden se encuentran estas limitantes?

En el testimonio citado, la médica A da cuenta de que la infertilidad como situación de padecimiento obtiene una respuesta médica, pero no porque se actúe sobre una imposibilidad biológica, sino porque la pareja logra materializar su deseo de tener una descendencia biológicamente vinculada. En muchos de los casos incluso no se logra un diagnóstico preciso de la imposibilidad de gestar. Esto constituye el diagnóstico de infertilidad sin causa aparente, el que, segundo Díniz y Gómez Costa (2002. p. 61)

[...] es anunciado toda vez que la causa de la infecundidad involuntaria no es localizada en el cuerpo de la mujer o del hombre en tratamiento por la medicina reproductiva. No hay señal o síntoma de esterilidad en el cuerpo: el único indicador de la enfermedad es la infecundidad involuntaria garantizada por la presuposición de la heterosexualidad de la pareja infértil. 
El diagnóstico de infertilidad sin causa aparente abre teóricamente la posibilidad de renegociar las TRA como un servicio potencialmente universal, no ligado a un cuerpo estéril (Diniz, Gómez Costa, 2006). Sin embargo la esterilidad como concepto continúa erigiéndose sobre razones sanitarias.

El discurso científico médico enfatiza las razones biológicas para la imposibilidad de obtener un embarazo y en este sentido construye una única sexualidad procreativa posible: la de la pareja heterosexual. Son estas parejas las que si no tuvieran un problema reproductivo lograrían la gestación. Esto asume que tales parejas tienen sexo coital y que éste se realiza con fines reproductivos - sin intermediar procedimientos o tecnologías anticonceptivas-, y posiciona a las TRA como herramientas que actúan frente a una situación no natural, ya que se asume que las parejas heterosexuales son naturalmente fértiles (Tamanini, 2009).

La alusión a la naturaleza como algo esencial sobre lo cual la biotecnología directamente actúa, en el sentido de destrabar lo que impide que la gestación se produzca y actuar replicando la potencialidad ideal del cuerpo (Strathern, 1995; Ramírez-Gálvez, 1999) resulta problemática a la hora de brindar atención a parejas homosexuales, mujeres sin pareja o sujetos en situaciones no consideradas médicamente patológicas en términos reproductivos.

Señalan Diniz y Gomez Costa (2006) que la presentación de las TRA como herramientas que buscan paliar un problema de salud reproductiva, definido en términos de infertilidad, ha sido parte de un proceso mediante el cual las TRA han buscado dejar atrás su identidad de herramientas destinadas a la producción de bebés para pasar a ser consideradas como biotecnologías que buscan sanar un problema reproductivo.

Pensadas como servicio las TRA deberían de brindarse a todos/as quienes pagaran por su asistencia y con el objetivo de dar un producto (bebé) con las características deseadas. En un contexto mercantil ${ }^{8}$ lo que las TRA producen es un bebé en tanto producto, ajustado al deseo de los clientes. En este orden de significación procedimientos como la selección sexual o de rasgos fenotípicos estarían más que avalados.

Presentar las TRA como un servicio permite a quienes trabajan en el área desplegar este tipo de intervenciones biotecnológicas que los y las especialistas saben que pueden generar resistencias culturales. También les obliga, por lo menos teóricamente, a contemplar como sujetos de asistencia a quienes no pueden ser considerados como imposibilitados de gestar por razones biológicas. Cada uno de estos movimientos encuentra sus límites culturales.

\footnotetext{
${ }^{8}$ La entrevista fue realizada antes que se aprobara la ley actualmente vigente.
} 
Si las TRA son concebidas en cambio como herramientas destinadas a solucionar un problema médico, deberían estar dirigidas solo a aquellos/as que se consideraran enfermos/as y con el objetivo de solucionar el padecimiento.

Si bien las clínicas rioplatenses han comenzado a brindar asistencia a sujetos o parejas que no entran en la definición de pareja infértil: ¿Ha cambiado la definición de infertilidad? ¿De qué nuevos sentidos se valen para permitirse incorporar a estos sujetos de atención que no entran en los cánones preestablecidos? ¿Cuáles son las resistencias que presentan los y las especialistas frente a estos cambios? ¿De qué órdenes culturales nos hablan tales resistencias?

Podemos enunciar dos órdenes que desde la medicina reproductiva rioplatense se definen como naturales y que en principio la práctica médicoreproductiva no debería trasgredir:

1. La naturaleza sexual del material reproductivo;

2. La naturaleza reproductiva del vínculo sexual.

Las situaciones que van en contra de estos órdenes no califican como situaciones de infertilidad en términos sanitarios, y por tanto presentan un escenario novedoso frente al cual la medicina reproductiva debe responder, ya sea generando un orden de sentido que las haga naturalmente atendibles $\mathrm{o}$ como situaciones imposibles de obtener respuesta por parte de las TRA.

\section{La naturaleza sexual del material reproductivo}

Médico B: [...] acá puede llegar una pareja de homosexuales y uno debería de atenderlos como a cualquiera. Pero me parece que entra en juego también otra cosa [...] Vienen porque quieren lograr un embarazo, ¿y quién puede lograr un embarazo? Puede lograr un embarazo la mujer que tiene útero, y tiene óvulos. La mujer que no tiene óvulos pero tiene útero si puede tener un embarazo, ahora la mujer que no tenga útero no va a quedar embarazada, se va a quedar embarazada otra persona y ya ese es un problema social no médico [...] Y lo que me asusta es que hay, digamos Florencias de la $V^{g}[\ldots]$ hay costumbres morales. Incluso para la propia gente que tiene esa condición es a no reconocer que tiene limitaciones como mujer y como hombre. (Argentina) ${ }^{10}$

Para este especialista los materiales reproductivos provenientes de un cuerpo biológicamente masculino no deberían considerarse como aporte para

\footnotetext{
${ }^{9}$ Se refiere a la travesti mediática argentina que tuvo uno hijo y una hija mediante vientre de alquiler con su pareja varón.

${ }^{10} \mathrm{En}$ las entrevistas en Argentina no se aclara tipo de institución porque son todos centros privados.
} 
la gestación de una futura criatura en tanto no son acordes a la identidad de género de quien demanda ser asistido/a.

La Florencia de la $\mathrm{V}$ a la que el técnico alude es una travesti argentina con identidad de género femenina para la cual es imposible aportar gametos reproductivos femeninos.

El límite moral del médico entrevistado se impone frente a lo que para él constituye el orden natural de lo reproductivo, en el cual un sujeto con identidad de género femenino debería aportar ovocitos. Para este especialista lo que en el caso de Florencia de la $\mathrm{V}$-como emblemático de una serie de casos similares reales o hipotéticos-, se está transgrediendo es un orden natural.

\section{La naturaleza reproductiva del vínculo sexual}

Como señalan Diniz y Gómez Costa (2006) la alusión a la infertilidad como una patología de pareja supone abordar la reproducción tecnológicamente mediada como una ayuda a la naturaleza y no como algo que rompe con el orden de lo natural. Advierten que al tomar como sujeto privilegiado de atención a la pareja estéril la medicina reproductiva se orienta a una moral destinada a preservar el orden sexual y productivo hetero-centrado que se supone obedece a razones naturales.

Esto es notorio en relación al tratamiento que la medicina reproductiva brinda a parejas heterosexuales que por diversas razones no quieren tener relaciones sexuales coitales pero sí descendencia biológica.

Médica A: Hay matrimonios no consumados. Yo tengo en el recuerdo ..., hay una que me viene a la mente porque siempre quiso tener tres hijos y nunca tuvo sexo. [...] A esa pareja yo le hice tres hijos. (Uruguay, Institución Mutual)

El deseo de procreación de estas parejas no es cuestionado porque está en consonancia con los arreglos socio sexuales y reproductivos; la posibilidad de lograr la reproducción está en la naturaleza de sus relaciones sexuales, en este caso no reales pero si presuntas. Como advierte Ramírez-Gálvez (2009), la intervención médica sobre la infecundidad involuntaria refuerza el papel de la medicina en la construcción de los significados referidos a la reproducción pero también en relación a la sexualidad. La medicina pasa a tener una función política de transmisión de comportamientos deseables en este contexto. El bio-poder penetra en estos cuerpos estableciendo un orden sexual deseable (Foucault, 1979).

En el caso de las parejas homosexuales las prácticas sexuales no tienen posibilidades biológicas de conducir a la reproducción, por lo cual quedan 
fuera de la definición de infertilidad como enfermedad reproductiva. Las TRA reproducen este orden negando el acceso a estas parejas o ubicándolas en la misma lógica que a la pareja heterosexual.

El relato de Sabrina -quien buscaba una descendencia biológicamente vinculada con su pareja mujer pero no estaba tomando medicación para ovular-, sobre la reacción del técnico que le hizo las ecografías para identificar el mejor momento para la inseminación con semen de donante es ilustrativo. El ecografista esperaba ver, en una mujer en proceso de reproducción asistida, un desarrollo folicular múltiple; su desconcierto al encontrar un solo folículo era tal que no lograba codificar lo que la imagen le mostraba,

Sabrina: [...] me empezó a preguntar qué medicación tomaba, con qué médico me atendía y no podía entender que yo no me estaba haciendo el protocolo [de infertilidad]. [...] El tipo no salía del asombro de que estaba todo bien, tenía dos ovarios, un útero y un folículo por explotar como sucede todos los meses.

El/la especialista que se enfrenta a situaciones de reproducción asistida está predispuesto a encontrarse siempre con una pareja biológicamente estéril. Esto ha sido parte de la constitución de la medicina reproductiva como una terapéutica cuyo objetivo es la solución de la infecundidad involuntaria a través de la medicalización de la reproducción (Diniz; Gómez Costa, 2006), proceso en el cual el protocolo de infertilidad tiene un lugar de significación central,

Entrevistadora: ¿Y parejas homosexuales han venido?

Médica D: Han venido pero son muy raras. [...] Hoy en día se hace inseminación con semen de donante... [...] se estudia, ¿verdad? [...] se estudia a la mujer que se va a hacer la inseminación porque tiene que estar apta para poder quedarse embarazada con una inseminación. [...] Tenemos una mujer de repente con las trompas tapadas; no va a poder quedar con la inseminación.

Entrevistadora: $O$ sea, se le hacen los estudios previos como a cualquier pareja.

Médica D: Como a cualquier pareja estéril. (Uruguay, Pereira Rossell)

El bio-poder (Foucault, 1979) actúa aquí tratando al cuerpo sexualmente desviado como si no lo fuera, como si cumpliera con la heteronormatividad prescripta. 


\section{El cuerpo femenino como cuerpo privilegiado de intervención}

Las TRA han ido, de manera paulatina, ampliando su campo de actuación. Uno de los mecanismos para ello ha sido la incorporación de nuevos sujetos de atención con las tensiones que antes desarrollaba. Otro ha sido la generación de una sensación de urgencia reproductiva que, más que habilita, obliga a las parejas pero fundamentalmente a las mujeres a acudir a la medicina reproductiva.

Esta urgencia reproductiva ha emergido como producto de una serie de condiciones: la creciente advertencia sobre las consecuencias negativa que sobre la capacidad reproductiva de las mujeres tiene el aplazamiento de la maternidad, el énfasis de las políticas reproductivas en la planificación familiar, y la maternidad en tanto condición simbólicamente central para la construcción de la femineidad.

El énfasis dado por la medicina reproductiva al factor femenino como elemento decisivo para el éxito de los tratamientos sumado a una cada vez más tardía edad reproductiva para las mujeres, ha llevado a las TRA a acotar el tiempo médicamente estimado para hablar de la existencia de una situación de infertilidad, pasando de dos años en la década de 1960 a definirse como plazo un año (Güezmes, 2005) en la actualidad.

Güezmes (2005) señala que además de la redefinición de este umbral temporal también han variado las situaciones que se considera requieren la intervención de la medicina reproductiva, incluyéndose entre otras a las mujeres que se embarazan pero sufren abortos espontáneos a repetición.

Esta urgencia reproductiva se enmarca asimismo en un marco político sanitario en el cual se ha trabajado fuertemente en la planificación de la reproducción ${ }^{11}$ con el objetivo de disminuir los embarazos no deseados así como el contagio de infecciones de transmisión sexual (ITS). Se trata de un escenario que se ha exacerbado a partir de la pandemia de VIH-Sida. Este énfasis en la planificación hizo suponer que toda la vida reproductiva podía ser controlada.

Catherina: [...] tengo amigas que dicen "vamos a buscar el año que viene”, porque este es chiquito, o para que nazca en la primavera. No tienen ni idea lo que es no saber si vas a tener algún día [...].

\footnotetext{
${ }^{11}$ Por detalles de estos programas en Uruguay ver Abracinskas y López Gómez (2006) y AUPF/RUDA (2008). En Argentina Programa Nacional de Educación Sexual y Procreación Responsable (2011).
} 
Las políticas centradas en la planificación y en la idea de relaciones sexuales protegidas han generado asimismo una visualización del cuerpo femenino como un cuerpo hiperfértil (Guthmann, 2005) que en el marco de una relación sexual no protegida conduciría inevitablemente al embarazo.

Claro que las mujeres entrevistadas relativizan esta idea de cuerpo hiperfértil reflexionando a partir de su experiencia,

Juliana: [...] una cosa que aprendí con esto y eso sí me quedó grabado a fuego, que con la planificación no... yo tenía tan estructurada la idea de que iba a tener un segundo embarazo fácil como el anterior, que lo iba a lograr rápido, y terminé con todo esto.

Marcia: El ginecólogo me explicaba que el ser humano es de los animales el menos fértil. Que en realidad las parejas que no tienen problemas tienen un 10 por ciento de probabilidad de quedar embarazada. Esos embarazos accidentales o el que busca y queda en el primer mes lo miro así, con una cara de envidia...

Esta dificultad de la especie humana para reproducirse, esta ineficiencia, es aludida también desde la medicina reproductiva como justificación para la actuación bio-tecnológica (Ramírez-Galvez, 2009).

Existe entonces un discurso proveniente de los organismos estatales, y materializado en las políticas de planificación familiar que coloca en la trama cultural la idea de que cualquier relación sexual no protegida conduce a un embarazo y otro, proveniente de la medicina reproductiva, centrado en las dificultades que existen para lograr y mantener una gestación, dificultad que aumenta en el contexto de determinadas circunstancias, una de las cuales es la edad de la mujer.

La necesidad esgrimida por la medicina reproductiva de actuar lo más rápidamente posible sobre los óvulos, sumado a la frustración de las parejas de no poder concretar un embarazo en un contexto socio histórico y cultural donde tal acontecimiento se presenta como rápidamente alcanzable, tiene dos consecuencias inmediatas sobre las prácticas médico-reproductivas y sobre el accionar de las mujeres/parejas para materializar el deseo de filiación biológica.

Por una parte, se incentiva a la consecución de acciones preventivas como la criopreservación de ovocitos, construyendo el deseo de maternidad como una entidad pronta a manifestarse cuando las condiciones estén dadas. En segundo lugar genera presión sobre el material reproductivo femenino, propiciando una cada vez más pronta intervención de ovocitos de donante en los procesos de alta complejidad. 
Como señala Guthmann (2005), el discurso de la medicina reproductiva legitima o impugna la instrumentalización del cuerpo o la explotación de algunas de sus partes para hacer posible ciertas intervenciones. El incentivo a utilizar óvulos de donante en los tratamientos de alta complejidad para garantizar el éxito de los mismos se acompaña a su vez de un discurso médico que asegura (simbólicamente) el vínculo filial con la madre a partir de lo que he dado en llamar la uterización del vínculo.

\section{Uterización del vínculo y disponibilidad para la ovorecepción}

La uterización del vínculo refiere al énfasis dado por la medicina reproductiva a la gestación y el proceso nutricio en el vientre materno para la ligación filial.

En su investigación sobre donación y recepción de gametos en Madrid, Alvarez Plaza (2008) destaca el carácter vinculante que a nivel simbólico posee la gestación. Sostengo que este carácter vinculante también es enfatizado por los y las especialistas rioplatenses. Esta garantía de vínculo depositada en la gestación amplía el margen de aceptación de material genético de otra mujerdonante.

Leticia, madre de mellizas con ovodonación, recalca el lugar del embarazo en su historia reproductiva. En el sitio de Internet "UruguayProcrea" escribía,

Una cosa thees q tener bien clara... el bebe q tengas sera hijo tuyo porq se formara en tu vientre, crecera en tu utero y nacera de ti... es tuyo!

Paralelamente al discurso centrado en el carácter vinculante de la gestación se ha desarrollado el interés por resguardar la participación biológica del varón de la pareja en el futuro ser. Este interés ha sido posible y al mismo tiempo ha justificado el avance de una nueva técnica: la ICSI.

La ICSI puede considerarse un ejemplo paradigmático de cómo los avances biotecnológico no son neutrales al género. A consecuencia de esta tecnología específica la ciencia puede producir un bebé sin recurrir a gametos masculinos de un donante pero obliga a pasar por un proceso de alta complejidad, en lugar de uno de baja complejidad que era lo que se debía de transitar en la mayoría de los casos que ahora se abordan con ICSI. Se trata así de preservar la filiación biológica masculina aún a costa de la mayor medicalización del cuerpo de la mujer, necesaria para un procedimiento de alta complejidad.

Los crecientes desarrollos en el campo de la medicina reproductiva no están orientados a preservar el cuerpo femenino. Si bien se está analizando 
la relación inversa entre mayor producción de ovocitos y mejor calidad ovocitaria, y por tanto se está estimulando mucho menos a las mujeres, la hiperestimulación se continúa favoreciendo por otros canales de significación cultural cómo la práctica de la ICSI.

El pasaje por la reproducción asistida para materializar el deseo del hijo biológicamente vinculado atinge así al cuerpo femenino y masculino de modos diferentes.

\section{Reflexiones finales}

La manera de presentar las TRA como una continuación en el orden de lo artificial de la dimensión de lo natural supone aceptar la construcción cultural de ambos dominios y sus fronteras. Estas fronteras también se han ido redefiniendo pero lo relevante a expresar es que siempre se presume un orden natural contra el cual las TRA no deberían accionar.

¿Dónde están las fronteras entre lo que se entiende natural y lo que se tiene por artificial en la práctica de la medicina reproductiva en el Río de la Plata?

Lo natural responde a un orden heterocentrado, ya que desde la medicina reproductiva el deseo que se habilita, por lo menos en términos de deseo natural, es aquel que va en el sentido de lo que biológicamente se define como infertilidad y que solo puede ser diagnosticado en una pareja infértil, y por tanto heterosexual, aunque como vimos no tenga relaciones sexuales coitales. Tampoco los casos diagnosticados como infertilidad sin causa aparente, que prometían ser un eslabón de cambio en la manera de pensar la infertilidad, sin ligarla a un cuerpo infértil (Diniz y Gómez Costa, 2006) han conducido a transformaciones en este sentido.

Le actuación de la medicina reproductiva en el Rio de la Plata reproduce así un orden fuertemente heterocentrado dando cuenta de formas de discriminación que aún persisten en la dimensión cultural. Tal reproducción no se define por la atención de la medicina reproductiva a parejas únicamente heterosexuales, sino por las formas en que se interviene a parejas no heterosexuales y los valores que se atribuye a los materiales reproductivos y al deseo de reproducción biológica de sujetos cuya identidad sexual no condice con su identidad de género.

A su vez los cuerpos biológicamente femeninos y masculinos y concomitantemente los materiales reproductivos provenientes de los mismos no son conceptualizados y abordados por la medicina reproductiva en términos equitativos y por tanto tampoco intervenidos de forma equivalente. En tal sentido la categoría analítica "género" resulta de particular relevancia 
para analizar los mecanismos de poder que imperan en el desarrollo e implementación de las TRA.

El cuerpo femenino es el que carga con la mayoría de los costos de las intervenciones biotecnológicas para lograr el hijo biológicamente vinculado. No solo en términos de una mayor medicalización porque la gestación todavía depende de un útero sino porque el desarrollo biotecnológico se orienta en su investigación y práctica, y es acompañado por una dimensión cultural que avala tal sentido, a intervenir mayormente el cuerpo femenino, y no solamente el de la mujer que busca tener descendencia mediante TRA sino también el de aquellas mujeres que comercian sus óvulos para estos tratamientos.

\section{Referências}

ABRACINSKAS, Lilián; LÓPEZ GÓMEZ, Alejandra. Monitoreo de políticas públicas en salud sexual y reproductiva desde la sociedad civil. II Encuentro universitario en salud, género y derechos sexuales y reproductivos. Avances en investigación nacional. Montevideo: Cátedra Libre en Salud Reproductiva, Sexualidad y Género (CATSR) Facultad de Psicología, Universidad de la República, 2006. p. 205-243.

ALTHABE, Gérard. Hacia una antropología del presente. Cuadernos de Antropología Social, v. 23, p. 13-34, 2006.

ALVAREZ PLAZA, Consuelo. La búsqueda de la eterna fertilidad: mercantilismo y altruismo en la donación de semen y óvulos. España: Alcalá Grupo Editorial, 2008.

AUPF/RUDA. Barreras: investigación y análisis sobre el acceso de las mujeres al derecho a decidir. Montevideo: Asociación Uruguaya de Planificación Familiar, 2008.

BARRÁN, José Pedro. Medicina y sociedad en el Uruguay del novecientos. Tomo I: El poder de curar y Tomo III: La invención del cuerpo. Montevideo: Banda Oriental, 1993.

BUTLER, Judith. O parentesco é sempre tido como heterossexual? Cadernos Pagu, v. 21, p. 219-260, 2003.

DINIZ, Debora; GÓMEZ COSTA, Rosely. Infertilidad e infecundidad: aceso a nuevas tecnologías reproductivas. In: Carlos F. Cáceres et al. (Orgs.). Sexualidad, estigma y derechos humanos: desafíos para el acceso a la salud en América Latina. Lima: Faspa/ UPCH, 2006. p. 55-67.

FOUCAULT, Michel. Historia de la sexualidad: la voluntad de saber. Buenos Aires: Siglo XXI Editores, 2002.

FOUCAULT, Michel. Microfísica del poder. Madrid: La Piqueta, 1979.

GEERTZ, Clifford. La interpretación de las culturas. Barcelona: Paidós, 1996.

GUBER, Rosana. El salvaje metropolitano. Buenos Aires: Paidós, 2004.

GÜEZMES, Ana. Las tecnologías de reproducción asistida: una aproximación desde la ética y las fugas feministas. SeriAs para el debate, v. 4, p. 24-51, 2005.

GUTHMANN, Yanina. El discurso bioético y la legitimación de las nuevas tecnologías reproductivas. SeriAs para el debate, n. 4, p. 90-111, 2005. 
MAYOBRE RODRÍGUEZ, Purificación. ¿Tiene género la Biotecnología? A propósito de los discursos de la maternidad en las Nuevas Tecnologías Reproductivas (N.T.R) $<$ http://webs.uvigo.es/pmayobre/indicedearticulos.htm\#biotecnologia> (22 oct. 2014).

OMS. Glosario de terminología en Técnicas de Reproducción Asistida (TRA). Versión revisada y preparada por el International Committee for Monitoring Assisted Reproductive Technology (ICMART) y la Organización Mundial de la Salud (OMS). Traducido y Publicado por la Red Latinoamericana de Reproducción Asistida, 2010 $<$ www.who.int/reproductivehealth/publications/infertility/art_terminology2/es/>.

PROGRAMA Nacional de Educación Sexual y Procreación Responsable. Políticas de Salud Sexual y Salud Reproductiva. Avances y desafíos <www.unfpaargentina.com. ar/sitio/archivos/originalbalancessyr.pdf $>$ (8 ago. 2013).

RAMÍREZ-GÁLVEZ, Martha Celia. Corpos fragmentados e domesticados na reprodução assistida. Cadernos Pagú, v. 33, p. 83-115, 2009.

RAMÍREZ-GÁLVEZ, Martha Celia. El uso de las tecnologías y la configuración de nuevas formas de reproducción. SeriAs para el Debate, v. n. 4, p. 80-89, 2005.

ROSTAGNOL, Susana. Representaciones y prácticas sobre sexualidad y métodos anticonceptivos entre hombres de sectores pobres urbanos. In: Sonnia Romero (Org.). Anuario antropología social y cultural en Uruguay 2002-2003. Montevideo: Nordam, p. 39-55, 2003.

SHORE, Cris. Virgin births and sterile debates: Anthropology and the New Reproductive Technologies. Current Anthropology, v. 33, n. 3, p. 295-314, 1992.

STRATHERN, Marilyn. Future Kinship and the study of culture. Revista Futures, V. 27 , n. 4 , p. 423-435, 1995.

STRATHERN, Marylin. Reproducing the future: essays on Anthropology kinship and the new reproductive technologies. Londres: Manchester University Press, 1992.

TAMANINI, Marlene. Reprodução assistida e gênero: o olhar das ciências humanas. Florianópolis: Editorial da UFSC, 2009.

Recebido em: 28 abr. 2014

Aprovado em: 12 jan. 2015

Autora correspondente:

Mariana Viera Cherro

Niteroi 987, entre Arizona y Rambla

Barra de Carrasco, Canelones

Uruguay 


\section{ANEXO \\ Mujeres entrevistadas}

\section{- Sandra}

- 43 años, realizó dos inseminaciones in vitro (asistencia mutual);

- Tres hijos/as: el primero producto de la primera inseminación in vitro, el segundo en un segundo procedimiento con óvulos congelados del procedimiento anterior y el tercero sin intervención de la medicina reproductiva;

- Cinco embarazos: un embarazo de abortó de manera voluntaria antes de los 30 y otro que abortó naturalmente antes de la segunda in vitro.

\section{- Milagros}

- 35 años, realizó una inseminación artificial y dos in vitro en Montevideo (clínica privada) y una in vitro en Buenos Aires (clínica privada);

- Dos hijos: gestados sin intervención tecnológica luego de haber decidido hacer una pausa en los tratamientos de reproducción asistida;

- Cuatro embarazos: además de los de su hijo e hija, tuvo un embarazo ectópico entre tratamiento y un embarazo con diagnóstico de "huevo muerto y retenido" que tuvo que abortar.

\section{P Juliana}

- 40 años, realizó una inseminación y una in vitro (asistencia mutual);

- Dos hijas: una hija de 12 años de su pareja anterior, sin intervención tecnológica, y una bebé de 10 meses resultado de una inseminación in vitro con su pareja actual;

- Dos embarazos.

\section{- Laura}

- 36 años, realizó cinco inseminaciones (asistencia mutual);

- Dos hijos: varones mellizos, resultado de la última inseminación;

- Uno embarazo.

\section{Camila}

- 43 años, realizó cuatro inseminaciones y tres in vitro, dos completas, de la última nacieron las mellizas y entre ambos tratamientos una in vitro que no llegó a completarse;

- Dos hijas: mellizas de la última in vitro;

- Uno embarazo.

\section{- Jazmín}

- 36 años, realizó tres inseminaciones, una in vitro con embriones frescos y una segunda in vitro con embriones congelados del primer tratamiento (asistencia mutual);

- Tres hijos: mellizos de 9 años del tratamiento in vitro con embriones congelados y un varón de 8 años gestado sin intervención de la medicina reproductiva;

- Dos embarazos. 


\section{$\rightarrow$ Andrea}

- 30 años, realizó dos inseminaciones y dos in vitro (mutual y privada);

- No tiene hijos;

- Uno embarazo: consecuencia de la segunda in vitro.

\section{- Marcia}

35 dos in vitro y una inseminación asistida (clínicas privadas en Montevideo y Buenos Aires);

- No tiene hijos;

- Uno embarazo: concebido de manera "natural" antes de la última inseminación. Concluyó en un aborto espontáneo.

\section{- Catherina}

- 34 años, realizó una inseminación asistida, una in vitro en Montevideo y otra en Buenos Aires;

- No tiene hijos;

- Ninguno embarazo.

\section{O Nirvana}

48 años, realizó varios tratamientos de reproducción asistida de baja complejidad (clínica privada);

- No tiene hijos;

- Ninguno embarazo.

\section{Leticia}

- 44 años, realizó un tratamiento in vitro con ovodonación en clínica pública (convenio con ASSE);

- Dos hijas: mellizas de un año y medio por inseminación in vitro con ovodonación;

- Dos embarazos: el de las mellizas y una gestación que ella terminó mediante un aborto voluntario muchos años antes de conocer al padre de las mellizas.

\section{Ingrid}

- 46 años, realizó solo estimulación hormonal;

- Cuatro hijas: cuatrillizas;

- Uno embarazo.

\section{- Sabrina}

- 32 años. Ella tiene una pareja mujer. El momento de la entrevista estaba embarazada a consecuencia de un tratamiento de baja complejidad, con semen de donante, realizado en Buenos Aires, donde no les hacen seguir el protocolo de infertilidad;

- No tiene hijos: está cursando el embarazo;

- Uno embarazo. 